\title{
Preparation and Analysis of Germanium Slices Having Prevalence of Either Serew or Edge Dislocations.
}

\author{
F. Calzecchi, A. Gardini and P. Gondi \\ Istituto di Fisica dell'Università - Bologna \\ Gruppo Nazionale di Struttura della Materia del CNR - Bologna
}

(Nuovo Cimento, $50 \mathrm{~B}, 263$ (1967))

The heading of Table II should be

TABLE II. - Percentages of the dislocation types appearing in the twisted and in the bent Ge samples. The data are averages relating to the various deformation degrees considered.

The heading of Table III should be

TABLE III. - Percentages of the dislocations lying along the directions specified in Fig. 1, in both twisted and bent specimens. The data refer to the highest deformation degrees, i.e., to a bending radius of $4 \mathrm{~cm}$ and to a torsion angle of $40^{\circ} \mathrm{cm}^{-1}$.

The percentages in Table III must be divided by two to sum up to 100 .

\section{Remarks on the Analysis of Statistical Reaction Spectra.}

\author{
E. Gadioli and L. Zetta \\ Istituto di Fisica dell'Università - Milano \\ Istituto Nazionale di Fisica Nucleare - Sezione di Milano \\ (Nuovo Cimento, 51 A, 1074 (1967))
}

This paper has been inadvertently published in Section A, although it pertains to Section B. 